\title{
Rational improvement of the engineered isobutanol-producing Bacillus subtilis by elementary mode analysis
}

\author{
Shanshan $\mathrm{Li}^{1}$, Di Huang ${ }^{1}$, Yong $\mathrm{Li}^{1}$, Jianping Wen ${ }^{1,2^{*}}$ and Xiaogiang Jia ${ }^{1,2}$
}

\begin{abstract}
Background: Isobutanol is considered as a leading candidate for the replacement of current fossil fuels, and expected to be produced biotechnologically. Owing to the valuable features, Bacillus subtilis has been engineered as an isobutanol producer, whereas it needs to be further optimized for more efficient production. Since elementary mode analysis (EMA) is a powerful tool for systematical analysis of metabolic network structures and cell metabolism, it might be of great importance in the rational strain improvement.

Results: Metabolic network of the isobutanol-producing B. subtilis BSUL03 was first constructed for EMA. Considering the actual cellular physiological state, 239 elementary modes (EMs) were screened from total 11,342 EMs for potential target prediction. On this basis, lactate dehydrogenase (LDH) and pyruvate dehydrogenase complex (PDHC) were predicted as the most promising inactivation candidates according to flux flexibility analysis and intracellular flux distribution simulation. Then, the in silico designed mutants were experimentally constructed. The maximal isobutanol yield of the LDH- and PDHC-deficient strain BSUL05 reached $61 \%$ of the theoretical value to $0.36 \pm 0.02 \mathrm{C}-\mathrm{mol}$ isobutanol/C-mol glucose, which was 2.3-fold of BSUL03. Moreover, this mutant produced approximately $70 \%$ more isobutanol to the maximal titer of $5.5 \pm 0.3 \mathrm{~g} / \mathrm{L}$ in fed-batch fermentations.

Conclusions: EMA was employed as a guiding tool to direct rational improvement of the engineered isobutanol-producing B. subtilis. The consistency between model prediction and experimental results demonstrates the rationality and accuracy of this EMA-based approach for target identification. This network-based rational strain improvement strategy could serve as a promising concept to engineer efficient $B$. subtilis hosts for isobutanol, as well as other valuable products.
\end{abstract}

Keywords: Rational strain improvement, Metabolic network, Elementary mode analysis, Target prediction, Bacillus subtilis, Isobutanol

\section{Background}

Isobutanol is considered as a leading candidate for the replacement of current fossil fuels [1,2]. Due to global environmental problems and fuel crises, isobutanol is expected to be produced in biotechnological process, which fulfills the demands of green and sustainable energy production [3]. Atsumi et al. [2] launched isobutanol bio-production in engineered Escherichia coli by harnessing the power of natural L-valine biosynthetic pathways. At present,

\footnotetext{
* Correspondence: jpwen@tju.edu.cn
'Department of Biological Engineering, School of Chemical Engineering and

*Correspondence: jpwen@tju.edu.cn
'Department of Biological Engineering, School of Chemical Engineering and Technology, Tianjin University, Tianjin 300072, China

${ }^{2}$ Key Laboratory of Systems Bioengineering, Ministry of Education, Tianjin ${ }^{2}$ Key Laboratory
300072 , China
}

\section{Biomed Central}

(C) 2012 Li et al.; licensee BioMed Central Ltd. This is an Open Access article distributed under the terms of the Creative Commons Attribution License (http://creativecommons.org/licenses/by/2.0), which permits unrestricted use, distribution, and reproduction in any medium, provided the original work is properly cited. isobutanol can be biosynthesized in several engineered microorganisms $[2,4-8]$.

As the best-characterized Gram-positive microorganism, Bacillus subtilis is regarded as a promising isobutanol producer owing to some valuable features. In addition to high isobutanol toxicity tolerance, $B$. subtilis has no significant codon usage bias, which facilitates the functional heterologous gene expression and pathway engineering. Besides, it can secrete several enzymes to depolymerize polysaccharides that are presented in large amounts in plant, and further utilize some resulted oligosaccharides and C5 sugar (e.g. L-arabinose) [9], which benefits isobutanol production from low-value feedstocks. So far, B. subtilis has 
been engineered for isobutanol production [6], whereas it still needs to be improved for higher yield.

Pathway modifications that direct metabolic flux towards the desired products play an important role in strain optimization. Several corresponding metabolic strategies, such as pathway reconstruction [10] and cofactor manipulation [11], have been well applied for metabolic evolution of the isobutanol producers. Nevertheless, these approaches are always time-consuming and subjected to laborious experiments for target validation. As cells are elaborate systems with highly interconnected metabolic networks, it is challenging to capture the full range of behaviors of a cell and identify the accurate targets for efficient strain improvement by analyzing a set of linear pathways.

To solve this problem, it is necessary to investigate the cell behaviors systematically. Current state-of-the-art omics technologies together with the next-generation sequencing promote the progress of systems biology, which allows the quantitative understanding of pathway operations during cellular metabolism by using the mutually related mathematical modeling and experiment [12]. This kind of framework addresses the questions of traditional metabolic engineering, accelerates the strain improvement process, and opens the door to a new era of network-based strain evolution [13-17]. Presently, several computational tools are developed for systematic cellular metabolism analysis [18]. Among all of them, elementary mode analysis (EMA) is acknowledged as a powerful tool to identify the metabolic network properties. Based on the nullspace and convex analysis, as well as the steady-state, EMA decomposes the complex metabolic network of a cell into a set of unique and indivisible pathways, which link all possible cellular physiological states $[19,20]$. Knowledge of these pathways allows the rational in silico design of an ideal host with specialized metabolic functionalities. In addition to previous applications for theoretical yield analysis and cellular phenotype prediction, EMA has drawn more and more attentions to develop efficient bioconversion platforms for the desired chemicals [13,15,21,22].

EMA is an attractive approach for strain improvement, whereas few attempts were performed in the isobutanolproducing microorganisms except for the recent cases implemented by Trinh et al. [23] and Matsuda et al. [24]. Furthermore, EMA has not been employed to explore the metabolic behaviors of $B$. subtilis until now. Based on the preceding successes, here we presented a network-based EMA strategy to rationally improve the engineered isobutanol-producing $B$. subtilis. First, the genome-scale metabolic network model of this strain was reconstructed and refined. Then, potential targets that influence isobutanol biosynthesis were identified, and the strain engineering strategy was proposed. Finally, the in silico designed isobutanol-producing $B$. subtilis mutants were experimentally constructed and further tested to verify the model prediction.

\section{Results}

Metabolic network analysis of the isobutanol-producing $B$. subtilis

Metabolic network of the isobutanol-producing B. subtilis BSUL03 for EMA comprises 131 reactions (36 reversible and 95 irreversible) and 132 metabolites (Additional file 1, Table S1 and Table S2). Overall, this metabolic network was decomposed into a total of 11,342 elementary modes (EMs). Each mode represents a unique possible pathway with balanced metabolites and cofactors. Among all the EMs, the majority are extreme modes exclusively linked to the production of either biomass or isobutanol, locating on the two axes of the plot (Figure 1). About $0.2 \%$ of the total EMs (25 EMs) allowed the maximal theoretical isobutanol yield of $0.67 \mathrm{C}$-mol isobutanol/C-mol glucose $(\mathrm{C}$-mol/C-mol), all of them had no biomass and byproduct. Conversely, only EMs without isobutanol biosynthesis could reach the maximal biomass yield of $0.52 \mathrm{C}$-mol biomass/C-mol glucose, close to the value of the wild type B. subtilis [25]. Considering the coupled formation of isobutanol and biomass, the total EMs were constrained to 2,216 EMs (less than $20 \%$ of the total EMs) with the maximal theoretical

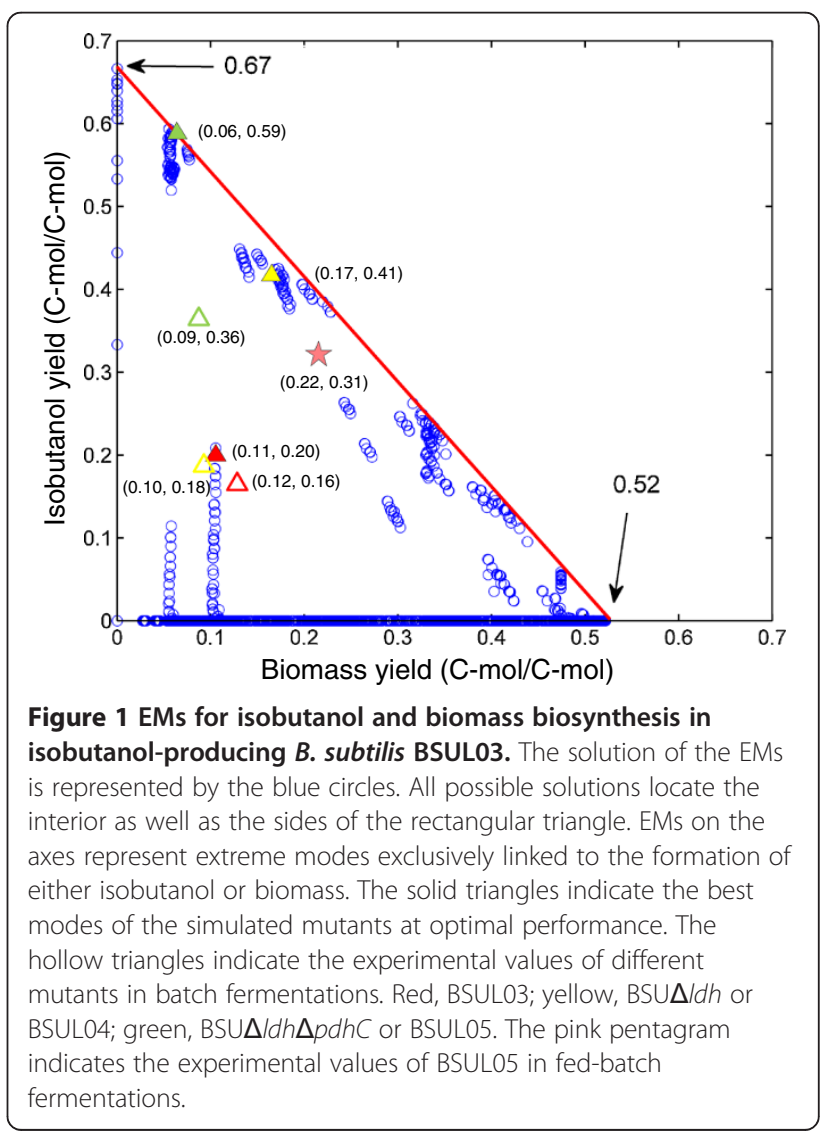


isobutanol yield of $0.64 \mathrm{C}$-mol/C-mol. The large number of EMs illustrates the robustness and flexibility of the cells to adapt themselves to particular conditions by using different pathways.

\section{Potential target identification}

EMA calculates the reaction flux and gives an extensive and profound insight into cell behaviors based on the metabolic network. Thus the potential bottlenecks could be investigated according to the flux distribution of EMs. For a precise prediction, EMs were classified and screened reasonably by taking the actual cellular physiological state into consideration. First, 2,216 EMs were generated by eliminating the extreme EMs from the total 11,342 EMs. Then, the unreasonable EMs (see Methods) were further excluded form 2,216 EMs, which finally resulted in 239 qualified EMs for the following analysis.

Statistical analysis was subsequently carried out. Flux correlation between each reaction and the objective reaction (R130) was scanned in silico. Reactions with valid flux correlation (linear regression coefficient $R^{2} \geq 0.7$ ) and statistical significance (by $t$-test) were selected for flux flexibility analysis, which reflects the influence of the given reaction on the objective reaction [22]. Here, 53 eligible reactions were obtained for flux flexibility analysis. For a given reaction, flux flexibility was analyzed by calculating the flux distribution among the qualified EMs and evaluated by the coefficient of standard deviation $(\mathrm{V} \sigma)$. Here, the top 12 candidates ranked via $V \sigma$ are listed in Table 1 , including pyruvate branches (R20, R22 and R43), 2ketoisovalerate (KIV) biosynthetic reactions (R59-R61), reactions involved in pentose phosphate pathway (PPP) (R31-R33, R36, R37) and one reaction responsible for redox balancing (R53). As shown in Table 1, reactions catalyzed by lactate dehydrogenase (LDH) and pyruvate

Table 1 Potential targets predicted by EMA based on flux correlation

\begin{tabular}{lllll}
\hline No. & Enzyme & EC No. & Gene & Vo \\
\hline R43 & lactate dehydrogenase & EC-1.1.1.27 & ldh & 2.76 \\
R22 & pyruvate dehydrogenase complex & EC-1.2.1.51 & pdhABCD & 1.65 \\
R59 & acetolactate synthase & EC-4.1.3.18 & alsS & 0.70 \\
R60 & ketol-acid reductoisomerase & EC-1.1.1.86 & ilvC & 0.70 \\
R61 & dihydroxy-acid dehydratase & EC-4.2.1.9 & ilvD & 0.70 \\
R53 & transhydrogenase & - & - & 0.57 \\
R36 & transketolase & EC-2.2.1.1 & tkt & 0.45 \\
R37 & transaldolase & EC-2.2.1.2 & ywjH & 0.44 \\
R31 & glucose 6-phosphate dehydrogenase & EC-1.1.1.49 & zwf & 0.39 \\
R32 & 6-phosphogluconolactonase & EC-3.1.1.31 & ykgB & 0.39 \\
R33 & phosphogluconate dehydrogenase & EC-1.1.1.44 & yqjl & 0.39 \\
R20 & pyruvate kinase & EC-2.7.1.40 & pyk & 0.35 \\
\hline
\end{tabular}

Detailed reactions are listed in Additional file 1, Table S1. dehydrogenase complex (PDHC) exhibited much higher flux flexibility than others. It suggests that the flux redistribution of the carbon drained off pyruvate is of great importance for isobutanol biosynthesis. Since both R43 and R22 showed the negative slope of linear regression with R130, LDH and PDHC were preliminarily selected as inactivation candidates for in vivo implementation in BSUL03.

To ensure the validity of the chosen targets, intracellular flux distributions of the parental strain BSUL03 and its corresponding mutants were further simulated. When considering lactate production, the theoretical isobutanol yield of BSUL03 sharply decreased from 0.64 to $0.20 \mathrm{C}-\mathrm{mol} / \mathrm{C}-\mathrm{mol}$ under the optimal conditions (Figure 1). Pyruvate node analysis showed that pyruvate flux split ratio of $\mathrm{LDH}$ to acetolactate synthease (ALS) was 3.7:1, much higher than the values of LDH to the other branches. As the effect of gene knockout could be simulated by removing the corresponding reactions from the stoichiometric matrix, the phenotype of the specific mutant could be analyzed by the remaining EMs. As shown in Figure 2, the relative flux of the suppositional LDH-deficient strain BSU $\Delta l d h$ was obviously changed in tricarboxylic acid (TCA) cycle, PPP and isobutanol biosynthetic pathway under the optimal conditions. Compared to BSUL03, the twofold relative flux through ALS of $\mathrm{BSU} \Delta l d h$ increased the theoretical isobutanol yield to $0.41 \mathrm{C}-\mathrm{mol} / \mathrm{C}$-mol (Figure 1). Additionally, pyruvate node analysis also showed that the flux drained off pyruvate was remarkably redistributed among the remaining branches. In particular, the flux fraction through pyruvate dehydrogenase complex (PDHC) was significantly increased by 10 -fold $(4.0 \% \rightarrow 44.3 \%)$, higher than the 1.5 -fold increase through ALS $(19.9 \% \rightarrow 49.4 \%)$. Synchronously, acetate secretion was enhanced by the excessive carbon flux through PDHC (Figure 2). In the suppositional LDH- and PDHC-deficient strain BSU $\Delta l d h \Delta p d h C$, ALS occupied $94 \%$ of pyruvate flux and became the dominant pyruvate gainer at optimal performance according to pyruvate node analysis. The relative flux through the objective reaction was approximately tripled in comparison with that of BSUL03 (Figure 2). As a result, this in silico strain obtained a 2 -fold increase of the theoretical isobutanol yield of $0.59 \mathrm{C}$-mol/C-mol (Figure 1).

As stated above, inactivation of LDH and PDHC could obviously redirect the pyruvate flux towards isobutanol biosynthetic pathway, and thus improve the theoretical yield. Therefore, LDH and PDHC were finally chosen for in vivo implementation.

\section{Experimental validation-I. Construction and characterization of the LDH-deficient isobutanol-producing $B$. subtilis}

According to the prediction, gene $l d h$ encoding for LDH was disrupted in BSUL03 by integrating plasmid pUCLKm (Additional file 2, Figure S1A) into the chromosome of 


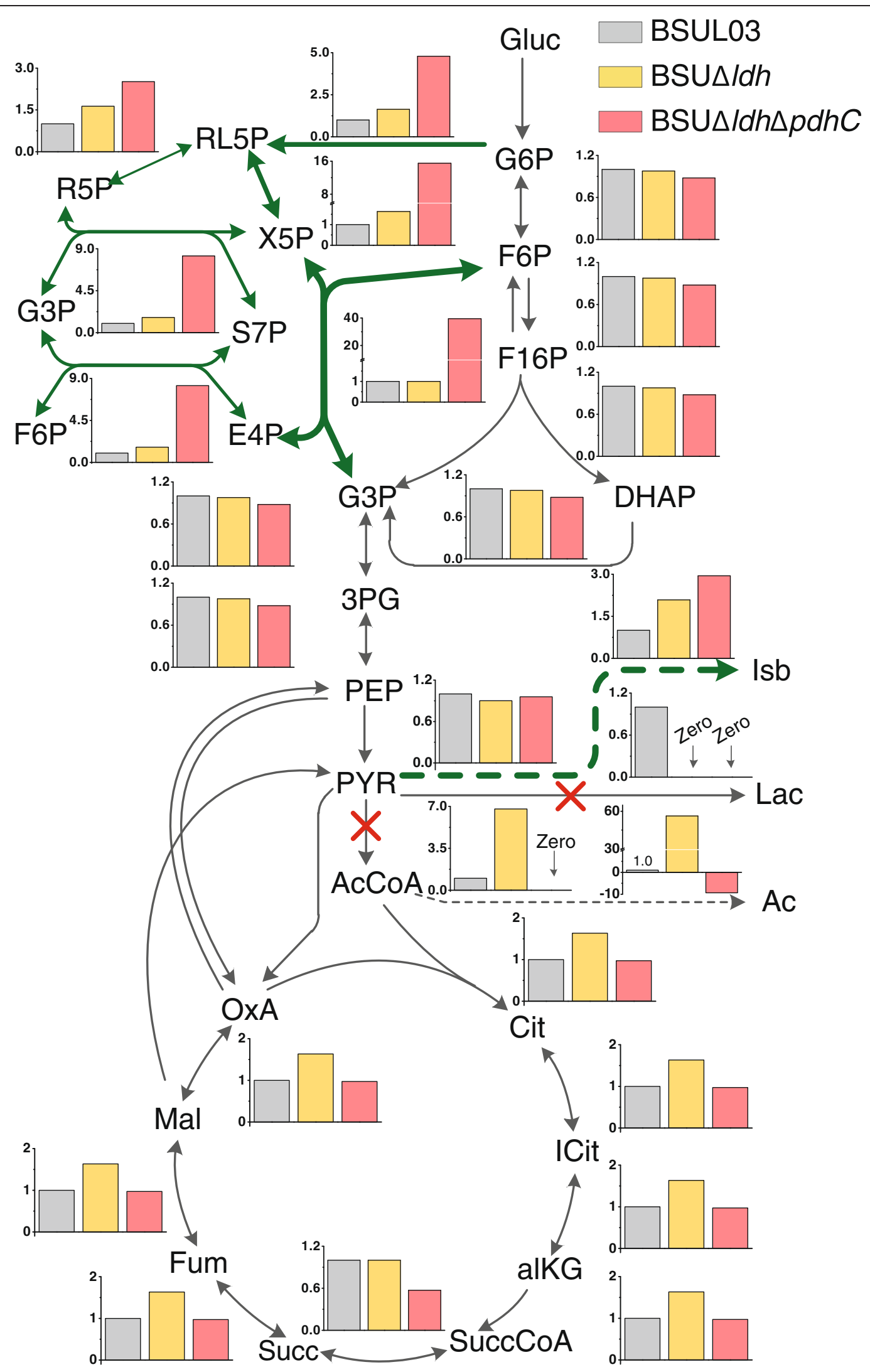

Figure 2 (See legend on next page.) 
(See figure on previous page.)

Figure 2 Simulated flux-fold changes of the central metabolism of isobutanol-producing B. subtilis under the optimal conditions. The chart represents the simulated fold change of the average flux through the central metabolism under optimal conditions considering the physiological states of different isobutanol-producing B. subtilis. All fluxes are given as relative molar flux normalized to 1 mol of glucose. Fold change of each reaction in the mutants is calculated by comparing to the corresponding flux in BSUL03. The dot lines indicate the multiple steps; the olive lines indicate the obviously increased flux by inactivating the targets (represented by red $x$ ); the negative flux indicates a reaction occurs in reverse direction. Metabolites abbreviations: Gluc, Glucose; G6P, D-Glucose 6-phosphate; F6P, D-Fructose 6-phosphate; F16P, D-Fructose 1,6bisphosphate; DHAP, Dihydroxyacetone phosphate; G3P, Glyceraldehyde 3-phosphate; 3PG, 3-Phospho-D-glycerate; PEP, Phosphoenolpyruvate; PYR, Pyruvate; AcCoA, Acetyl-coenzyme A; Cit, Citrate; ICit, Isocitrate; alKG, 2-Oxoglutarate; SuccCoA, Succinyl-CoA; Succ, Succinate; Fum, Fumarate; Mal, L-Malate; OxA, Oxaloacetate; RL5P, Ribulose-5-phosphate; R5P, alpha-D-Ribose 5-phosphate; X5P, Xylulose-5-phosphate; G3P, Glyceraldehyde 3-phosphate; S7P, Sedoheptulose 7-phosphate; E4P, D-Erythrose 4-phosphate; Isb, Isobutanol; Lac, L-Lactate; Ac, Acetate.

BSUL03. Recombinants resistant to kanamycin were selected for PCR confirmation by using a pair of primers $l d h-\mathrm{F}$ and $l d h-\mathrm{R}$. BSUL03 showed a $0.9 \mathrm{~kb}$ PCR band, while the $l d h$-disrupted strain BSUL04 showed a $2 \mathrm{~kb}$ PCR band (Additional file 2, Figure S1B). Assay of enzyme activity showed that LDH activity of BSUL04 was $0.06 \pm 0.01$ $\mathrm{U} / \mathrm{mg}$, while that was $5.20 \pm 0.06 \mathrm{U} / \mathrm{mg}$ of BSUL03. These results indicated that LDH activity was destroyed in BSUL04.

Strain BSUL04 showed a $21 \%$ and $25 \%$ lower biomass $(1.62 \pm 0.03 \mathrm{~g} / \mathrm{L})$ and specific growth rate $\left(0.29 \pm 0.01 \mathrm{~h}^{-1}\right)$ than BSUL03 (Figure 3), respectively, suggesting that cell growth was impaired by $l d h$ disruption. In microaerobic batch fermentations, BSUL04 produced $2.11 \pm 0.15 \mathrm{~g} / \mathrm{L}$ isobutanol with a yield of $0.18 \pm 0.02 \mathrm{C}$-mol/C-mol, which was $12.5 \%$ higher than that of BSUL03. As expected, lactate was undetected in BSUL04, however, ethanol increased by $67 \%$ to $1.82 \pm 0.27 \mathrm{~g} / \mathrm{L}$ and acetate even tripled to $10.65 \pm 1.04 \mathrm{~g} / \mathrm{L}$ (Table 2). Combining with the almost unchanged intracellular pyruvate pool (Figure 4), these phenomena implied that the carbon flux originally

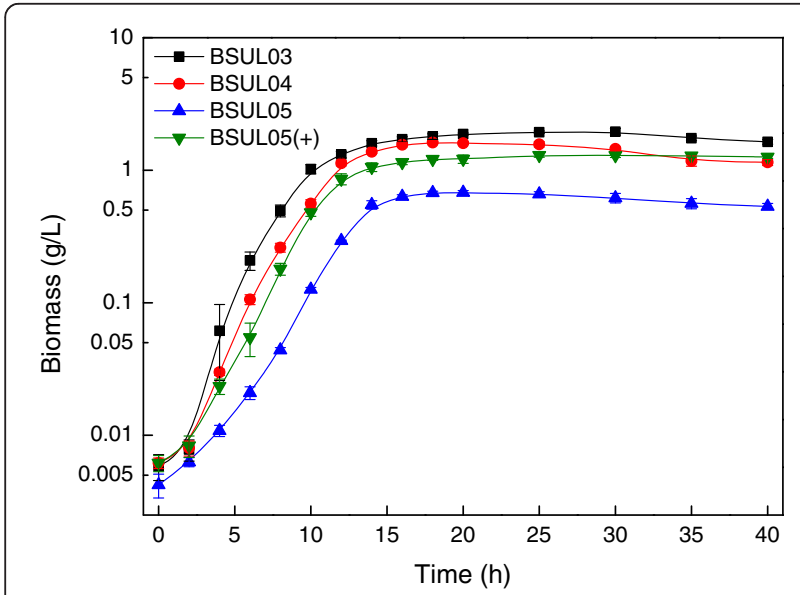

Figure 3 Cell growth comparison of different isobutanolproducing $B$. subtilis. The experiments were carried out in LBGSM-I medium under microaerobic conditions. The plus symbol indicates that the strain was cultivated in the medium supplemented with $3 \mathrm{~g} / \mathrm{L}$ sodium acetic acid. consumed by LDH was mainly splitted by the competing branches via PDHC and isobutanol biosynthetic pathway via ALS in BSUL04, in agreement with pyruvate node analysis.

\section{Experimental validation-II. Construction and characterization of the LDH- and PDHC-deficient isobutanol-producing $B$. subtilis}

In $B$. subtilis, the nonpolar PDHC-deficient mutants can be obtained by the interruptions of any gene involved in $p d h A B C D$ operon except for the essential gene $p d h A$ [26]. As the core of PDHC, the E2 subunits encoded by $p d h C$ affect PDHC activity most significantly. Therefore, $p d h C$ was reasonably selected for gene knockout in the present work. Recombinants with kanamycin and tetracycline resistance suggested that $p d h C$ were deleted by integrating the plasmid pUCPTet (Additional file 2, Figure S1C) into the chromosome of BSUL04 via double homologous recombinant. By using a pair of primers $p d h C 1-\mathrm{F}$ and $p d h C 2-\mathrm{R}$, the band of BSUL04 and the pdhC-disrupted B. subtilis BSUL05 were 2 and $2.75 \mathrm{~kb}$, respectively (Additional file 2, Figure S1D). PDHC activity of BSUL03 and BSUL04 were $0.31 \pm 0.01 \mathrm{U} / \mathrm{mg}$ and $0.27 \pm 0.01 \mathrm{U} / \mathrm{mg}$, respectively, whereas it could not be detected in BSUL05, implying that PDHC was inactivated in BSUL05.

Strain BSUL05 exhibited a longer transition time to exponential phase: $4 \mathrm{~h}$ compared to $2 \mathrm{~h}$ in BSUL03 and BSUL04. Besides, biomass $(0.68 \pm 0.02 \mathrm{~g} / \mathrm{L})$ and the specific growth rate $\left(0.17 \pm 0.02 \mathrm{~h}^{-1}\right)$ were $35 \%$ and $44 \%$ of BSUL03, respectively (Figure 3 ). Along with the suppressed cell growth, isobutanol production and intracellular ATP concentration of BSUL05 sharply decreased to $1.17 \pm 0.12 \mathrm{~g} / \mathrm{L}$ and $96 \pm 7 \mathrm{nM}$, respectively. Meanwhile, a conspicuous increase of intracellular pyruvate concentration (approximately by 50 -fold) was also observed (Figure 4). Satisfactorily, lactate, acetate and ethanol were undetected in fermentation broth (Table 2). In comparison with BSUL04, BSUL05 doubled isobutanol yield to $0.36 \pm 0.02 \mathrm{C}-\mathrm{mol} / \mathrm{C}-\mathrm{mol}$, which was $61 \%$ of the predicted value $(0.59 \mathrm{C}-\mathrm{mol} / \mathrm{C}-\mathrm{mol})$. Though cell growth 
Table 2 Comparison of metabolic profiles of different isobutanol-producing B. subtilis under microaerobic conditions

\begin{tabular}{llllll}
\hline Strain & $\begin{array}{l}\text { Isobutanol production } \\
(\mathbf{g} / \mathbf{L})\end{array}$ & $\begin{array}{l}\text { Isobutanol yield } \\
(\mathbf{C}-\mathbf{m o l} / \mathbf{C}-\mathbf{m o l})\end{array}$ & $\begin{array}{l}\text { Lactate } \\
\mathbf{( g / L )}\end{array}$ & $\begin{array}{l}\text { Acetate } \\
(\mathbf{g} / \mathbf{L})\end{array}$ & $\begin{array}{l}\text { Ethanol } \\
(\mathbf{g} / \mathbf{L})\end{array}$ \\
\hline BSUL03 & $1.95 \pm 0.18$ & $0.16 \pm 0.01$ & $3.67 \pm 022$ & $3.73 \pm 0.29$ & $1.09 \pm 0.13$ \\
BSUL04 & $2.11 \pm 0.15$ & $0.18 \pm 0.02$ & $\mathrm{ND}$ & $10.65 \pm 1.04$ & $1.82 \pm 0.27$ \\
BSUL05 & $1.17 \pm 0.12$ & $0.36 \pm 0.02$ & $\mathrm{ND}$ & $\mathrm{ND}$ & $\mathrm{ND}$ \\
BSUL05(+) & $2.28 \pm 0.17$ & $0.29 \pm 0.01$ & $\mathrm{ND}$ & $4.46 \pm 0.85$ \\
\hline
\end{tabular}

Strains were cultured in LBGSM-I medium for $40 \mathrm{~h}$ under microaerobic conditions. The plus symbol indicates medium for BSUL05 cultivation is supplemented with $3 \mathrm{~g} / \mathrm{L}$ sodium acetic acid; ND represents the product is not detected.

and isobutanol production were inhibited in BSUL05, both of them could be well restored by external acetate addition (Table 2). Simultaneously, the intracellular ATP concentration increased by $180 \%$ to $270 \pm 18 \mathrm{nM}$, and the intracellular pyruvate concentration decreased by 95\% to $2.6 \mathrm{mM}$ (Figure 4). However, an unexpected net acetate accumulation was noticed during fermentations (Table 2).

\section{Isobutanol biosynthesis profile of $B$. subtilis BSUL05}

Strain BSUL05 showed an improved isobutanol biosynthetic capability in batch fermentations. Here it was further assessed in fed-batch fermentations. At the same time, the parental strain BSUL03 was taken as control. As shown in Figure 5, both strains grew exponentially under the aerobic conditions. Though glucose and acetate were almost exhausted during this period, little isobutanol was detected in the fermentation broth. After entering the oxygen-limited period, cell growth of the two strains slowed down, whereas isobutanol biosynthesis began to accelerate. Despite of the almost identical cell growth tendency, the two strains presented different isobutanol biosynthetic behaviors. Isobutanol titer of
BSUL03 reached the peak $(3.2 \pm 0.4 \mathrm{~g} / \mathrm{L})$ around $50 \mathrm{~h}$ and declined thereafter, whereas that of BSUL05 continuously increased to $5.5 \pm 0.3 \mathrm{~g} / \mathrm{L}$ at the end of fermentations. Meanwhile, isobutanol yield was up to $53 \%$ of the theoretical value to $0.31 \pm 0.02 \mathrm{C}$ - $\mathrm{mol} / \mathrm{C}$-mol, which was 1.9-fold of BSUL03. Contrastively, the final acetate concentration of BSUL05 decreased to $0.2 \mathrm{~g} / \mathrm{L}$, which was merely about $10 \%$ of that of BSUL03. These results demonstrate the better isobutanol biosynthetic performance of the rational improved BSUL05 than the parental strain.

\section{Discussion}

Some progress is being made in strain improvement of the engineered isobutanol producers $[2,4,6]$. However, these strategies are always time-consuming and laborious due to the incomplete understanding of the complex cellular behaviors. Network-based EMA is believed to be an attractive approach to handle this problem, while the relevant investigations are rather limited in isobutanol producers. For that reason, the EMA-based design strategy was first employed as a guiding tool to tailor the

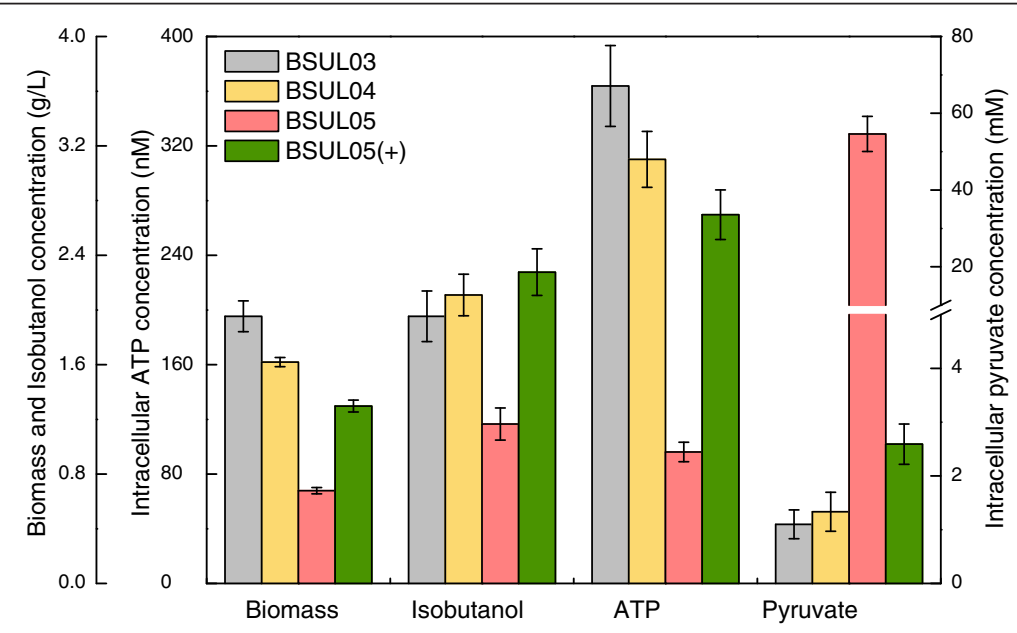

Figure 4 Relationships of cell growth, isobutanol production, intracellular ATP and pyruvate of different isobutanol-producing $B$. subtilis. The experiments were carried out in LBGSM-I medium under microaerobic conditions. The plus symbol indicates that the strain was cultivated in the medium supplemented with $3 \mathrm{~g} / \mathrm{L}$ sodium acetic acid. 


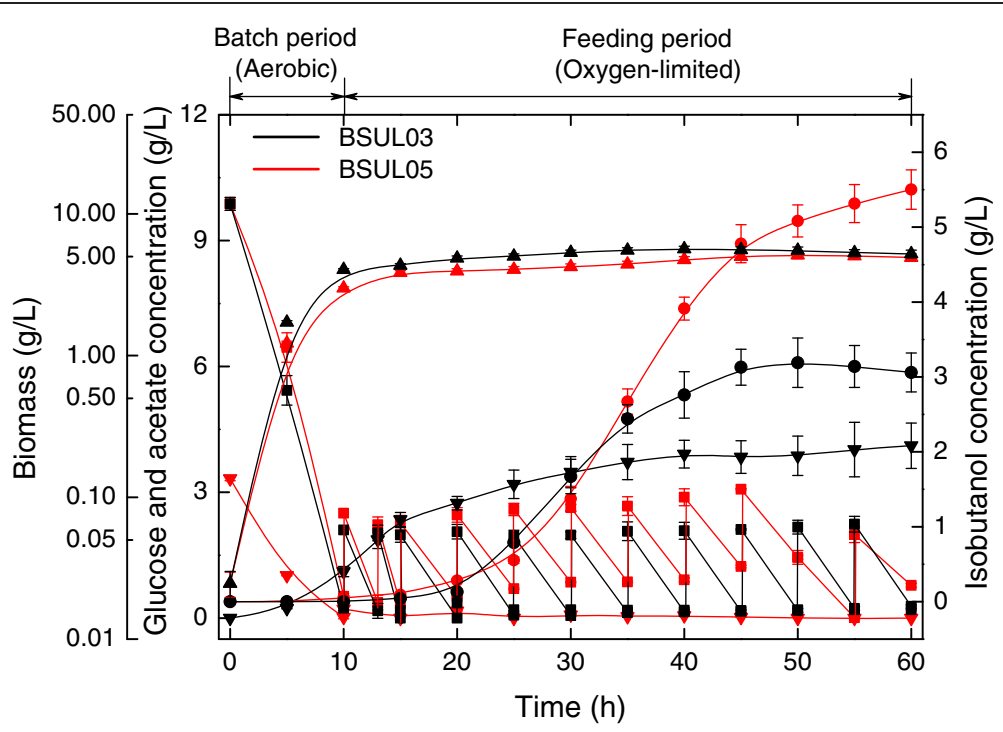

Figure 5 Comparison of metabolic profiles of BSUL03 and BSUL05 in fed-batch fermentations. Time-course profiles of cell growth, isobutanol, glucose and acetate in fed-batch fermentations were compared between BSUL05 and the parental strain BSUL03. Symbols: Square, glucose; circle, isobutanol; triangle, biomass; downtriangle, acetate.

engineered B. subtilis for better isobutanol-producing performance [6].

EMA decomposes a metabolic network into a set of unique and non-divisible pathways that represent all possible physiological states of the cells. Different from algorithms such as MOMA [27] and OptGene [28] that could identify only one optimal solution, EMA calculates all the possible optimal pathway solutions. It offers an opportunity to investigate the flux distribution at different performances, comprehend the underlying cellular behaviors, and draft the blueprint for strain improvement.

For a precise prediction, the qualified EMs should be chosen from the total EMs, as only those conformed to the real cellular physiological state are meaningful to strain optimization. Here, two points should be taken into account. One is that the extreme EMs without the synchronous formation of isobutanol and biomass need to be eliminated. The other one is that the central metabolism is active under the oxygen-limited condition despite the expression of the involved genes are downregulated [11,29]. Therefore, EMs with non-positive carbon flux through glycolysis and the irreversible reactions of PPP and TCA cycle are unreasonable and also need to be excluded. Previous reports showed that linear relationship existed between fluxes through the target candidates and the objective reaction $[22,30]$. Therefore, the statistically significant reactions were picked out for further analysis. Both flux flexibility analysis (Table 1) and intracellular flux simulation (Figure 2) proposed LDH and PDHC as the most promising inactivation targets. This fully coincides with the fact that both LDH $\left(K_{m}=3.0 \mathrm{mM}\right)[31]$ and PDHC
$\left(K_{m}=4.3 \mathrm{mM}\right)$ [32] possess much higher pyruvate affinity than ALS $\left(K_{m}=13.6 \mathrm{mM}\right)$ [33] in B. subtilis. In addition, LDH can be activated in an FNR (a transcriptional activator for anaerobically induced genes) independent manner under oxygen-limited conditions [29]. Following $l d h$ and $p d h \mathrm{C}$, alsS, ilvC and ilvD involved in KIV biosynthetic reactions were identified as potential amplification targets (Table 1), holding an identical view with the previous findings that overexpression of these genes is beneficial to isobutanol production enhancement $[2,5,6]$. Apart from the above targets, potential candidates also included a transhydrogenase and five PPP-related encoding genes, implying that the intracellular redox state maintains close ties with isobutanol biosynthesis.

As analyzed above, the corresponding mutants were experimentally constructed to validate the EMA prediction. Disruption of $l d h$ eliminated lactate and increased isobutanol yield by $12.5 \%$, meaning that the carbon flux is indeed redirected towards isobutanol as simulated. Although the observations were consistent with the findings from several experimental studies $[4,5]$, the yield increment was not so much as predicted. We speculated that this could be primarily put down to the undesirably drastic increase of acetate, which is a well-known inhibitor in fermentation. Romero et al. [34] demonstrated that, in B. subtilis, LDH plays a pivotal role in maintaining the redox equilibrium $\left(\mathrm{NAD}(\mathrm{P}) \mathrm{H} / \mathrm{NAD}(\mathrm{P})^{+}\right)$under fermentative conditions. As ketol-acid reductoisomerase and alcohol dehydrogenase for isobutanol biosynthesis require NADPH and NADH as cofactor, respectively, the discrepancy might also be ascribed to the depressed enzyme activity, which was relevant to the disturbed 
intracellular redox state caused by $l d h$ disruption. This conjecture agreed with the speculation about the intimate connection between cellular redox state and isobutanol biosynthesis during target identification. Further disruption of $p d h C$ obviously doubled isobutanol yield to $0.36 \pm 0.02 \mathrm{C}$-mol/C-mol, and decreased acetate and ethanol to an undetectable level (Table 2). These data suggested that, on one aspect, PDHC inactivation triggered a significant carbon flux shift, which favors isobutanol biosynthesis and was confirmed by intracellular metabolites analysis (unpublished data). On another aspect, byproduct elimination prevented the broth from overacidification and increased isobutanol yield [35]. Therefore, EMA is accurate enough to predict the targets and guide the rational improvement of the engineered isobutanol-producing B. subtilis.

When taking glucose as the sole carbon source, both cell growth and isobutanol production were suppressed in BSUL05 (Figure 3 and Table 2). As shown in Figure 1, EMA reveals an inverse relationship between the yield of isobutanol and biomass owing to the shared precursor competition $[15,22]$. Thus, it was plausible to observe that strain with higher isobutanol biosynthetic efficiency exhibited a lower cell growth. Furthermore, the impaired cell growth of this PDHC-deficient strain might also be accounted for the lack of a pivotal building block, acetylcoenzyme A (AcCoA). As for the decreased isobutanol production, it might be explained by two reasons. On one hand, an appropriate biomass is necessary for the production of desired products, so the lower isobutanol production of BSUL05 might be attributed to the impaired cell growth. On the other hand, the depressed TCA cycle caused by AcCoA shortage led to ATP scarcity (Figure 4), which influenced cell growth and normal expression of the enzymes for isobutanol biosynthesis. Delightedly, we found that external acetate addition could increase the intracellular ATP concentration and restore the impaired cell growth along with isobutanol production (Figure 4). These results turned out that acetate could not only serve as a carbon source, but also as an energy source in BSUL05. Unexpectedly, a net acetate accumulation was observed, which might be relevant to the putative pyruvate oxidase (POX) in B. subtilis [36]. Originally, the small amount of acetate biosynthesized via POX could be assimilated via acetyl-CoA synthetase, whereas this kind of balance might be destroyed by the accelerated metabolism induced by external acetate addition. Fortunately, acetate could be well controlled at a low concentration in fed-batch fermentations (Figure 5), implying the acetate productionconsumption equilibrium could be balanced under appropriate conditions.

The rational improved isobutanol-producing B. subtilis BSUL05 displayed much better isobutanol biosynthetic performances than the parental strain BSUL03 both in batch and fed-batch fermentations (Table 2 and Figure 5). However, the maximal isobutanol yield of BSUL05 obtained in the present work $(0.36 \pm 0.02 \mathrm{C}$-mol/C-mol $)$ was $39 \%$ lower than the in silico prediction (0.59 C-mol/C-mol) (Figure 1). Such a discrepancy was also noticed by other researchers [15]. It is inferred that this phenomenon might be explained by the following reasons. First, EMA revealed that efficient isobutanol biosynthesis depends on the low oxygen level (data not shown), whereas the precise control of the oxygenlimited condition is really a challenge $[4,23]$. Then, as EMA hinted the important role of cellular redox state in isobutanol biosynthesis (Table 1 and Figure 2), this discrepancy might partially ascribe to the disturbed redox equilibrium caused by $l d h$ and $p d h C$ deletion [4,5]. Next, isobutanol biosynthesis might be inhibited by some negative effects of the cell regulatory system that was not considered by EMA. Besides, some unknown regulation mechanisms could also interpret the fact. Finally, as the 'just-in-time' gene transcription (and the associated enzyme expression) shows clear influence on efficient bioproduction $[37,38]$, the lower isobutanol yield of the mutant might be attributed to the uncoordinated isobutanol biosynthetic pipelines, which could not be explored by the metabolic network. Therefore, the aforementioned factors need to be investigated in future studies to construct a more efficient isobutanol-producing B. subtilis. At these points, the improved performance of BSUL05 has confirmed that EMA is a valuable tool for rapid and precise target identification to engineer $B$. subtilis with stronger isobutanol biosynthetic capability. This metabolic network-based rational strain improvement strategy could be applied to construct valuable industrial $B$. subtilis hosts for the production of isobutanol and other desired products.

\section{Conclusions}

In this work, we presented the first report on rational improvement of the isobutanol-producing B. subtilis by employing an EMA-based strategy. Flux flexibility analysis and in silico simulation predicted LDH and PDHC as the most promising inactivation targets, which were further validated experimentally. The maximal isobutanol yield and titer of the mutant BSUL05 reached 2.3- and 1.7-fold of BSUL03 to $0.36 \pm 0.02 \mathrm{C}$ - $\mathrm{mol} / \mathrm{C}$ - $\mathrm{mol}$ and $5.5 \pm 0.3 \mathrm{~g} / \mathrm{L}$, respectively, showing a stronger isobutanol biosynthetic capability. The consistency between model prediction and experimental results demonstrates that EMA is a reliable approach for target identification and strain optimization. Moreover, EMA dropped a hint of the close relationship between isobutanol biosynthesis and cellular redox state, which provides a valuable insight into further improvement of the isobutanol-producing B. subtilis. Our results demonstrate that the EMA-based prediction could serve 
as a useful strategy to guide strain engineers towards improved bio-production in B. subtilis, as well as other microorganisms.

\section{Methods \\ Metabolic network reconstruction}

The genome-scale metabolic network of isobutanolproducing $B$. subtilis was constructed by introducing the isobutanol biosynthetic reactions, which allows the conversion from KIV to isobutanol, into the previously described B. subtilis 168 network [39]. For EMA, the model was further refined. Except for the central carbon metabolism, linear reactions involved in other subsystems were lumped when necessary (Additional file 1, Table S1). It takes glucose as the sole carbon source via phosphotransferase system, and contains other substrates ammonium, sulfur and oxygen. Products of isobutanol, lactate, acetate, ethanol, valine, carbon dioxide and biomass, as well as ATP are considered as external metabolites. For ATP production in the respiratory chain, a $\mathrm{P} / \mathrm{O}$ ratio of 1.33 (for $\mathrm{NADH}$ ) and 0.89 (for $\mathrm{FADH}_{2}$ ) was assumed [40]. The biomass composition and cell molecular were taken from Dauner and Sauer [41]. Water, protons and phosphate were assumed to be ubiquitous and unlimited in the cells.

\section{EMA}

EMA was implemented by METATOOL [42]. The script files and compiled shared library of METATOOL 5.1 were downloaded from the METATOOL website (http:// www.biozentrum.uni-wuerzburg.de/bioinformatik/). EMA results were analyzed using Excel Microsoft Corp. for mode sorting and filtering.

\section{Potential target identification based on flux correlation}

For a given elementary flux mode $j$, the relative metabolic flux $\left(\mathrm{v}_{i, j}\right)$ of each reaction $i$ is normalized to the glucose uptake flux. The yield $\left(\mathrm{Y}_{P / C, \mathrm{j}}\right)$ of biomass and isobutanol was calculated according to Eq. 1.

$$
\mathrm{Y}_{\mathrm{P} / \mathrm{C}, \mathrm{j}}=\frac{\mathrm{S}_{\mathrm{p}, \mathrm{j}}}{\mathrm{S}_{\mathrm{C}, \mathrm{j}}} \frac{\xi_{\mathrm{p}, \mathrm{j}}}{\xi_{\mathrm{C}, \mathrm{j}}}, 1 \leq \mathrm{j} \leq \mathrm{n}
$$

Here, $\mathrm{S}$ stands for the EMs matrix with the dimension of $j \times i$, where $i$ and $j$ are the number of reactions and EMs, respectively. The symbol $\xi$ represents the molar carbon content expressed in C-mol per mol biomass or isobutanol.

To investigate whether a reaction $i$ is a potential target, a chosen set of EMs was screened for further statistical analysis by considering the cellular physiological state of isobutanol biosynthesis. First, the extreme EMs were excluded from the total EMs. Then, EMs with nonpositive carbon flux through glycolysis and the irreversible reactions of PPP and TCA cycle were unreasonable and further eliminated, generating the qualified EMs for statistical analysis. The linear flux correlation between the reaction $i$ and R130 was primarily analyzed. Here, a cut-off value of the regression coefficient $R^{2}=0.7$ was set for preliminary target selection [22]. If the linear slope is positive, the reaction $i$ is regarded as the amplification target, otherwise it is the gene knockout candidate. Besides, the statistical significance of these targets was also examined by $t$ test, which was a criterion to classify the qualified reactions for flux flexibility analysis. Flux flexibility reflects the influence of a given reaction $i$ on the objective reaction. It is analyzed by calculating the flux distribution among the qualified EMs and measured by $\mathrm{V} \sigma$ as described in Eq. 2. Thus, the potential target could be ranked via the value of Vo.

$$
\mathrm{V} \sigma_{i}=\frac{\delta_{v_{i}}}{v_{i}}, 1 \leq \mathrm{i} \leq \mathrm{m}
$$

Here, $\delta_{v_{i}}$ and $\overline{v_{i}}$ refer to the deviation and the average flux of reaction $i$, respectively. The variable $m$ refers to the number of reactions in the metabolic network.

\section{Microbial strains and media}

All strains and plasmids used in this work are listed in Table 3. All Bacillus mutants used in this work were derivatives of BSUL03 [6]. E. coli JM109 was used to propagate all plasmids. Unless stated otherwise, E. coli and B. subtilis were cultured in Luria-Bertani (LB) medium (peptone $10 \mathrm{~g} / \mathrm{L}$, yeast extract $5 \mathrm{~g} / \mathrm{L}$, and sodium chloride $5 \mathrm{~g} / \mathrm{L}$ ) at $37{ }^{\circ} \mathrm{C}$. Batch fermentations were carried out in LBGSM-I medium (LB medium supplemented with glucose $20 \mathrm{~g} / \mathrm{L}, \mathrm{K}_{2} \mathrm{HPO}_{4} 2 \mathrm{~g} / \mathrm{L}, \mathrm{KH}_{2} \mathrm{PO}_{4}$ $1 \mathrm{~g} / \mathrm{L}$ and $10^{3}$ dilution of Trace Metal Mix A5 [2]). Fedbatch fermentations were carried out in LBGSM-III medium (identical to LBGSM-I except for glucose $10 \mathrm{~g} /$ L). Glucose feeding solution (glucose $500 \mathrm{~g} / \mathrm{L}$ ) and acetate feeding solution (sodium acetic acid $200 \mathrm{~g} / \mathrm{L}$ ) were used as feeding stocks during fed-batch fermentations. Antibiotics were added appropriately as follows: ampicillin $100 \mu \mathrm{g} / \mathrm{mL}$, spectinomycin $100 \mu \mathrm{g} / \mathrm{mL}$, kanamycin $10 \mu \mathrm{g} / \mathrm{mL}$ and tetracycline $20 \mu \mathrm{g} / \mathrm{mL}$.

\section{Gene cloning and plasmid construction}

All oligonucleotides used in this work are listed in (Additional file 3 Table S3). Standard techniques for nucleic acid manipulation were used as described by Sambrook et al. [43]. By using genomic DNA of B. subtilis BSUL03 as template, the ldh gene (code for LDH) [GenBank: 938348] was amplified with a pair of primers $l d h-\mathrm{F}$ and $l d h-\mathrm{R}$, the homologous arms $p d h C 1$ and $p d h C 2$ of pdhC (code for PDHC E2 subunit) [GenBank: 936010] were amplified with two pairs of primers $p d h C 1-\mathrm{F}$ and $p d h C 1-\mathrm{R}, p d h C 2-\mathrm{F}$ and $p d h C 2-\mathrm{R}$, respectively. The $X b a \mathrm{I}-$ 
Table 3 Strains and plasmids used in this study

\begin{tabular}{|c|c|c|}
\hline Name & Relevant genotype & Source \\
\hline \multicolumn{3}{|l|}{ Strains } \\
\hline E. coli JM109 & recA1, endA1, gyrA96, thi-1, hsdR17, supE44, relA1, $\Delta($ lac-proAB)/F'traD36, proAB+, laclq, lacZ $\Delta M 15]$ & TransGen Biotech \\
\hline BSUL03 & $\Delta a m y E::\left(\mathrm{P}_{43}:: k i v d-a d h 2\right), \mathrm{P}_{43}: .: i / v D-i l v C-a l s S_{;} \mathrm{Spc}^{r}, \mathrm{Em}^{r}$ & [6] \\
\hline BSUL04 & BSUL03 with lactate dehydrogenase inactivation $(\Delta / d h) ; \mathrm{Spc}^{r}, \mathrm{Em}^{r}, \mathrm{Km}^{r}$ & This study \\
\hline BSUL05 & BSUL04 with pyruvate dehydrogenase complex E2 subunit inactivation $(\Delta / d h \Delta p d h C) ; \mathrm{Spc}^{r}, \mathrm{Em}^{r}, \mathrm{Km}^{r}$, $\mathrm{Tet}^{r}$ & This study \\
\hline \multicolumn{3}{|l|}{ Plasmids } \\
\hline pUC18 & E. coli cloning vector; Ampr & Laboratory stock \\
\hline pDK & B. subtilis integration vector with amyE locus; $\mathrm{Amp}^{r}, \mathrm{Km}^{\mathrm{r}}$ & BGSC [50] \\
\hline pHY300PLK & E. coli-B. subtilis cloning vector; Ampr, Tet ${ }^{r}$ & Takara \\
\hline pUCL & pUC18 containing Idh fragment of B. subtilis, Amp ${ }^{r}$ & This study \\
\hline pUCLKm & pUCL containing kanamycin resistance cassettes from pDK; $\mathrm{Amp}^{r}, \mathrm{Km}^{\mathrm{r}}$ & This study \\
\hline pUCP01 & pUC18 containing homology arm pdhC1 fragment of B. subtilis; Amp ${ }^{r}$ & This study \\
\hline pUCP02 & pUCP01 containing homology arm pdhC2 fragment of B. subtilis; Amp ${ }^{r}$ & This study \\
\hline pUCPTet & pUCP02 containing tetracycline resistance cassettes from pHY300PLK; Ampr, Tet $^{r}$ & This study \\
\hline
\end{tabular}

XmaI digested $l d h$ PCR product was cloned into pUC18 cut with the same enzymes to create pUCL. Then the kanamycin-resistant cassette cut from plasmid pDK with EcoRI and EcoRV was blunted and ligated into the plasmid pUCL cut with EcoRV, creating pUCLKm (Additional file 2, Figure S1A). Plasmid pUCP01 was obtained by cloning the HindIII-PstI digested $p d h C 1$ PCR product into pUC18 cut with the same enzymes. Then the BamHI-EcoRI digested $p d h C 2$ PCR product was cloned into pUCP01 cut with the same enzymes, creating pUCP02. The tetracycline-resistant cassette was amplified by using plasmid PHY300PLK as template with a pair of primers Tet-F and Tet-R. The Pst $\mathrm{I}-$ BamHI digested PCR product was then cloned into pUCP02 cut with the same enzymes, creating pUCPTet (Additional file 2, Figure S1C).

\section{Construction of isobutanol-producing $B$. subtilis mutants}

To obtain B. subtilis mutants, the integration vectors pUCLKm and pUCPTet were sequentially transformed into BSUL03 cells by using the competent cell method [44]. B. subtilis recombinants were selected by kanamycin resistance or/and tetracycline resistance, and further confirmed by PCR using two pairs of primers $l d h-\mathrm{F} / l d h$ $\mathrm{R}$ and $p d h C 1-\mathrm{F} / p d h C 2-\mathrm{R}$, respectively. The LDH mutant and the $\mathrm{LDH}$ and $\mathrm{PDHC}$ mutant were designated as BSUL04 and BSUL05, respectively.

\section{Cultivation}

Pre-cultures were prepared by cultivating one fresh colony in the liquid LB medium at $240 \mathrm{rpm}$ for $8 \mathrm{~h}$. The $1 \%(\mathrm{v} / \mathrm{v})$ inoculation was adopted in all experiments. Batch fermentations for phenotype growth and metabolic profile assays were carried out in $500 \mathrm{~mL}$ screw-cap flasks with LBGSM-
I medium under the microaerobic conditions (40\% work volume, $200 \mathrm{rpm}, 37^{\circ} \mathrm{C}$ ) for $40 \mathrm{~h}$. Fed-batch fermentations were performed in $400 \mathrm{~mL}$ LBGSM-III cultures in a fedbatch Pro fermentation system (DASGIP, Germany) under two-stage (aerobic/oxygen-limited) conditions for $60 \mathrm{~h}$. Dissolved oxygen was measured by an oxygen electrode (Mettler Toledo, Germany). For aerobic conditions (0$10 \mathrm{~h}$ ), it was controlled at $30 \pm 1 \%$ of saturation in a cascade by stirring from 200 to $700 \mathrm{rpm}$ with 1 volume of air per volume of medium per minute (vvm). For oxygenlimited conditions $(10-60 \mathrm{~h}$ ), it was adjusted to $5 \pm 1 \%$ by reducing the stirrer and aeration to $50 \mathrm{rpm}$ and $0.5 \mathrm{vvm}$, respectively. The $\mathrm{pH}$ adjusted by $2 \mathrm{M} \mathrm{NaOH}$ and $2 \mathrm{M} \mathrm{HCl}$ was maintained at 7.0 by a standard $\mathrm{pH}$ electrode (Mettler Toledo, Germany). Foam was prohibited by manual injection of appropriate antifoamer (Sigma 204). When glucose concentration fell below $1 \mathrm{~g} / \mathrm{L}, 1.6 \mathrm{~mL}$ of glucose feeding solution was added. For PDHC-deficient strains, sodium acetic acid were originally supplemented into the medium at a final concentration of $3 \mathrm{~g} / \mathrm{L}$ and $3.4 \mathrm{~g} / \mathrm{L}$ in batch and fed-batch fermentations, respectively. Besides, $0.4 \mathrm{~mL}$ of acetate feeding solution was added coupled with glucose feeding solution during feeding period. All the fermentative experiments were performed in triplicate.

\section{Enzyme activity assays}

Cells for $\mathrm{LDH}$ and PDHC enzyme activity assays were grown at $37^{\circ} \mathrm{C}$ in $25 \mathrm{~mL} \mathrm{LB}$ medium, harvested at the exponential phase $(6 \mathrm{~h})$ and the beginning of the stationary phase $(14 \mathrm{~h})$, respectively, washed twice with cold potassium phosphate buffer (100 mM, pH 7.0), and then suspended in $5 \mathrm{~mL}$ of the same buffer. Cell extracts were obtained by sonication (UH-250A, Autoscience 
instrument Co., Ltd.; $40 \mathrm{k} \mathrm{Hz}, 10$-s pulse and 5-s intervals, total $10 \mathrm{~min})$ and centrifugation $(17,900 \mathrm{~g}$ for $10 \mathrm{~min}$ at $4^{\circ} \mathrm{C}$ ). The supernatant was kept on ice until determination. The standard assay for LDH activity (towards lactate) was spectrophotometrically (340 nm) monitored at $25^{\circ} \mathrm{C}$ by following the oxidation of $\mathrm{NADH}$ [45]. The activity of the PDHC was performed as described by Murarka et al. [46]. Total protein concentrations were measured by Bradford assay [47].

\section{Measurement of intracellular ATP concentration}

The intracellular ATP concentration was measured by the ATP assay kit (Beyotime, China). Cells $\left(1 \times 10^{4}\right)$ harvested at the mid-log phase were mixed with $20 \mu \mathrm{L}$ lysis buffer and homogenized by vortex, and then centrifuged at $15,300 \mathrm{~g}$ for $5 \mathrm{~min}$ at $4^{\circ} \mathrm{C}$. Other procedures such as lysis buffer preparation, background luminescence correction and ATP measurement were performed as the ATP Assay protocol (Beyotime). The emitted light by luminescent reaction was quantified by a luminometer (Synergy H4 Hybrid Microplate reader, BioTek, USA). ATP concentrations of the samples were calculated from the standard curve using linear regression (0.5-50 nM). Each experiment was carried out in triplicate and each sample was measured five times.

\section{Intracellular pyruvate analysis}

To analyze the intracellular pyruvate, $5 \mathrm{~mL}$ mid-log phase cell suspension was harvested using vacuum filtration (AP-01P Vacuum Pump, Tianjin Auto Science Co., Ltd.; cellulose nitrate, $0.22 \mu \mathrm{m}$ pore size) and washed three times each with $20 \mathrm{~mL} 0.9 \%$ cold $\mathrm{NaCl}$ solution (the whole filtration procedure including the washing was finished in less than $30 \mathrm{~s}$ ) [48]. Metabolite extraction and sample preparation were carried out as described [49]. Sampling was carried out five times in parallel for each experiment.

Intracellular pyruvate analysis was implemented by liquid chromatography-tandem quadrupole mass spectrometry (Bruker MicroTOF-Q II, Germany) equipped with a $250 \times 4.6 \mathrm{~mm}$ aminopropyl column (Luna $\mathrm{NH}_{2}, 5 \mu \mathrm{m}$ particle size, Phenomenex). The detailed separation and elution conditions, as well as data analysis approaches were carried out as described previously [49].

\section{Analytical methods}

Cell concentration was determined by measuring the optical density of culture broth at $600 \mathrm{~nm}\left(\mathrm{OD}_{600}\right)$. Biomass was calculated by multiplying $\mathrm{OD}_{600}$ by a conversion factor of 0.325 . The quantitative analysis of glucose and fermentation metabolites (alcohols, organic acids and other compounds) were also performed as before [6].

\section{Additional files}

Additional file 1: Metabolic network model of the engineered isobutanol-producing B. subtilis BSUL03. Details of model construction are described in Additional file 1. Stoichiometric equations involved in the model for EMA are listed in Table S1. All abbreviations of metabolites are listed in Table $\mathbf{S 2}$.

Additional file 2: Plasmids construction and gene knockout confirmation. Plasmids used for gene disruption of $/ d h(A)$ and $p d h C(C)$. PCR confirmation of gene knockout for $l d h$ (B) and $p d h C(D)$. The genomic DNA of BSUL03 was used as control. Lane M, 1 kb DNA ladder; Lane $\mathrm{S}$, the positive double crossover mutant; Lane + , the positive control; Lane -, the negative control.

Additional file 3: Oligonucleotides used in this study.

\section{Abbreviations}

EMA: elementary mode analysis; EMs: elementary modes; LDH: lactate dehydrogenase; PDHC: pyruvate dehydrogenase complex; ALS: acetolactate synthease; KIV: 2-ketoisovalerate; POX: pyruvate oxidase; AcCoA: acetylcoenzyme A; EMP: glycolysis pathway; PPP: pentose phosphate pathway; TCA cycle: tricarboxylic acid cycle; LB: Luria-Bertani.

\section{Competing interests}

The authors declare that they have no competing interests.

\section{Authors' contributions}

JPW and SSL conceived and designed the research. SSL performed the main experiments, the statistical analysis and drafted the manuscript. DH participated in model simulation and manuscript revision. YL performed mutant construction and enzyme assays. XQJ contributed to metabolites analysis. JPW supervised the research and revised the manuscript. All authors read and approved the final manuscript.

\section{Acknowledgements}

The authors appreciate the kind donation of plasmid pDK from Dr. Danier R. Zeigler and the Bacillus Genetic Stock Center (BGSC), The Ohio State University. This research was financially supported by the National 973 Project of China (No. 2007CB714302), the Key Program of National Natural Science Foundation of China (Grant No. 20936002), National Natural Science Foundation of China (No. 20976124), Specialized Research Fund for the Doctoral Program of Higher Education (20110032130005) and the Programme of Introducing Talents of Discipline to Universities (No. B06006).

Received: 23 March 2012 Accepted: 13 July 2012

Published: 3 August 2012

\section{References}

1. Dellomonaco C, Fava F, Gonzalez R: The path to next generation biofuels: successes and challenges in the era of synthetic biology. Microb Cell Fact 2010, 9:3.

2. Atsumi S, Hanai T, Liao JC: Non-fermentative pathways for synthesis of branched-chain higher alcohols as biofuels. Nature 2008, 451:86-U13.

3. Fava F, Ohtake H, Pesaresi P: Biotechnology for a more sustainable environment decontamination and energy production. J Biotechnol 2012, 157:443-445.

4. Blombach B, Riester T, Wieschalka S, Ziert C, Youn JW, Wendisch VF, Eikmanns BJ: Corynebacterium glutamicum tailored for efficient isobutanol production. Appl Environ Microbiol 2011, 77:3300-3310.

5. Smith KM, Cho KM, Liao JC: Engineering Corynebacterium glutamicum for isobutanol production. Appl Microbiol Biotechnol 2010, 87:1045-1055.

6. Li SS, Wen JP, Jia XQ: Engineering Bacillus subtilis for isobutanol production by heterologous Ehrlich pathway construction and the biosynthetic 2-ketoisovalerate precursor pathway overexpression. Appl Microbiol Biotechnol 2011, 91:577-589.

7. Higashide W, Li YC, Yang YF, Liao JC: Metabolic engineering of Clostridium cellulolyticum for production of isobutanol from cellulose. Appl Environ Microbiol 2011, 77:2727-2733.

8. Chen X, Nielsen KF, Borodina I, Kielland-Brandt MC, Karhumaa K: Increased isobutanol production in Saccharomyces cerevisiae by overexpression of genes in valine metabolism. Biotechnol Biofuels 2011, 4:21. 
9. Stülke J, Hillen W: Regulation of carbon catabolism in Bacillus species. Annu Rev Microbiol 2000, 54:849-880.

10. Atsumi S, Wu TY, Eckl EM, Hawkins SD, Buelter T, Liao JC: Engineering the isobutanol biosynthetic pathway in Escherichia coli by comparison of three aldehyde reductase/alcohol dehydrogenase genes. Appl Microbiol Biotechnol 2010, 85:651-657.

11. Bastian S, Liu X, Meyerowitz JT, Snow CD, Chen MMY, Arnold FH: Engineered ketol-acid reductoisomerase and alcohol dehydrogenase enable anaerobic 2-methylpropan-1-ol production at theoretical yield in Escherichia coli. Metab Eng 2011, 13:345-352.

12. Cassman M, Arkin A, Doyle F, Katagiri F, Lauffenburger D, Stokes C: Systems Biology. Springer Verlag: International Research and Development; 2007.

13. Becker J, Zelder O, Häfner S, Schröder H, Wittmann C: From zero to hero-Design-based systems metabolic engineering of Corynebacterium glutamicum for L-lysine production. Metab Eng 2011, 13:159-168.

14. Park JH, Lee KH, Kim TY, Lee SY: Metabolic engineering of Escherichia coli for the production of L-valine based on transcriptome analysis and in silico gene knockout simulation. P Natl Acad Sci USA 2007, 104:7797-7802.

15. Unrean P: Trinh CT. Srienc F: Rational design and construction of an efficient E. coli for production of diapolycopendioic acid. Metab Eng 2010, 12:112-122.

16. Brochado AR, Matos C, Møller BL, Hansen J, Mortensen UH, Patil KR: Improved vanillin production in baker's yeast through in silico design. Microb Cell Fact 2010, 9:84.

17. Asadollahi MA, Maury J, Patil KR, Schalk M, Clark A, Nielsen J: Enhancing sesquiterpene production in Saccharomyces cerevisiae through in silico driven metabolic engineering. Metab Eng 2009, 11:328-334.

18. Ruppin E, Papin JA, de Figueiredo LF, Schuster S: Metabolic reconstruction, constraint-based analysis and game theory to probe genome-scale metabolic networks. Curr Opin Biotechnol 2010, 21:502-510

19. Schuster S, Fell DA, Dandekar T: A general definition of metabolic pathways useful for systematic organization and analysis of complex metabolic networks. Nat Biotechnol 2000, 18:326-332.

20. Trinh CT, Wlaschin A, Srienc F: Elementary mode analysis: a useful metabolic pathway analysis tool for characterizing cellular metabolism. Appl Microbiol Biotechnol 2009, 81:813-826.

21. Driouch $H$, Melzer $G$, Wittmann $C$ : Integration of in vivo and in silico metabolic fluxes for improvement of recombinant protein production. Metab Eng 2012, 14:47-58.

22. Melzer G, Esfandabadi ME, Franco-Lara E, Wittmann C: Flux Design: In silico design of cell factories based on correlation of pathway fluxes to desired properties. BMC Syst Biol 2009, 3:120.

23. Trinh CT, Li J, Blanch HW, Clark DS: Redesigning Escherichia coli metabolism for anaerobic production of isobutanol. Appl Environ Microbiol 2011, 77:4894-4904.

24. Matsuda F, Furusawa C, Kondo T, Ishii J, Shimizu H, Kondo A: Engineering strategy of yeast metabolism for higher alcohol production. Microb Cell Fact 2011, 10:70

25. Sauer U, Hatzimanikatis V, Hohmann HPGM, Manneberg M, van Loon AP, Bailey JE: Physiology and metabolic fluxes of wild-type and riboflavinproducing Bacillus subtilis. Appl Environ Microbiol 1996, 62:3687-3696.

26. Gao HC, Jiang X, Pogliano K, Aronson Al: The E1 beta and E2 subunits of the Bacillus subtilis pyruvate dehydrogenase complex are involved in regulation of sporulation. J Bacteriol 2002, 184:2780-2788.

27. Segrè $D$, Vitkup $D$, Church GM: Analysis of optimality in natural and perturbed metabolic networks. P Natl Acad Sci USA 2002, 99:15112-15117.

28. Patil KR, Rocha I, Förster J, Nielsen J: Evolutionary programming as a platform for in silico metabolic engineering. BMC Bioinformatics 2005, 6:308

29. Nakano MM, Zuber P: Anaerobic growth of a "strict aerobe" (Bacillus subtilis). Annu Rev Microbiol 1998, 52:165-190.

30. Choi HS, Lee SY, Kim TY, Woo HM: In Silico Identification of Gene Amplification Targets for Improvement of Lycopene Production. Appl Environ Microbiol 2010, 76:3097-3105.

31. Garvie El: Bacterial lactate dehydrogenases. Microbiol Mol Biol Rev 1980, 44:106-139.

32. Hodgson JA, Lowe PN, Perham RN: Wild-type and mutant forms of the pyruvate dehydrogenase multienzyme complex from Bacillus subtilis. Biochem J 1983, 211:463-472.

33. Atsumi S, Li Z, Liao JC: Acetolactate synthase from Bacillus subtilis serves as a 2-ketoisovalerate decarboxylase for isobutanol biosynthesis in Escherichia coli. Appl Environ Microbiol 2009, 75:6306-6311.
34. Romero S, Merino E, Bolívar F, Gosset G, Martinez A: Metabolic engineering of Bacillus subtilis for ethanol production: lactate dehydrogenase plays a key role in fermentative metabolism. Appl Environ Microbiol 2007, 73:51905198.

35. Jian J, Zhang SQ, Shi ZY, Wang W, Chen GQ, Wu QO: Production of polyhydroxyalkanoates by Escherichia coli mutants with defected mixed acid fermentation pathways. Appl Microbiol Biotechnol 2010, 87:2247-2256.

36. Petersohn A, Antelmann H, Gerth U, Hecker M: Identification and transcriptional analysis of new members of the $\sigma^{B}$ regulon in Bacillus subtilis. Microbiology 1999, 145:869-880.

37. Medema MH, Breitling R, Bovenberg R, Takano E: Exploiting plug-and-play synthetic biology for drug discovery and production in microorganisms. Nat Rev Microbiol 2011, 9:131-137.

38. Liu S, Zhang DX, Wang M, Cui WJ, Chen KK, Du GC, Chen J, Zhou ZM: The order of expression is a key factor in the production of active transglutaminase in Escherichia coli by co-expression with its propeptide. Microb Cell Fact 2011, 10:112

39. Oh YK, Palsson BO, Park SM, Schilling CH, Mahadevan R: Genome-scale reconstruction of metabolic network in Bacillus subtilis based on highthroughput phenotyping and gene essentiality data. J Biol Chem 2007, 282:28791-28799.

40. Sauer U, Bailey JE: Estimation of P-to-O ratio in Bacillus subtilis and its influence on maximum riboflavin yield. Biotechnol Bioeng 1999, 64:750-754.

41. Dauner M, Sauer U: Stoichiometric growth model for riboflavin-producing Bacillus subtilis. Biotechnol Bioeng 2001, 76:132-143.

42. Pfeiffer T, Sánchez-Valdenebro I, Nuño JC, Montero F, Schuster S: METATOOL: for studying metabolic networks. Bioinformatics 1999, 15:251-257.

43. Sambrook J: Russel DW: Molecular cloning: a laboratory manual. 3rd edition. Cold Spring Harbor, NY: Cold Spring Harbor Laboratory Press; 2001

44. Anagnostopoulos C, Spizizen J: Requirements for transformation in Bacillus subtilis. J Bacteriol 1961, 81:741-746.

45. Savijoki K, Palva A: Molecular genetic characterization of the L-lactate dehydrogenase gene (IdhL) of Lactobacillus helveticus and biochemical characterization of the enzyme. Appl Environ Microbiol 1997, 63:2850-2856.

46. Murarka A, Clomburg JM, Moran S, Shanks JV, Gonzalez R: Metabolic analysis of wild-type Escherichia coli and a pyruvate dehydrogenase complex (PDHC)-deficient derivative reveals the role of PDHC in the fermentative metabolism of glucose. J Biol Chem 2010, 285:31548-31558,

47. Bradford MM: A rapid and sensitive method for the quantitation of microgram quantities of protein utilizing the principle of protein-dye binding. Anal Chem 1976, 72:248-254.

48. Bolten CJ, Kiefer P, Letisse F, Portais JC, Wittmann C: Sampling for metabolome analysis of microorganisms. Anal Chem 2007, 79:3843-3849.

49. Wang ZW, Chen T, Ma XH, Shen Z, Zhao XM: Enhancement of riboflavin production with Bacillus subtilis by expression and site-directed mutagenesis of zwf and gnd gene from Corynebacterium glutamicum. Bioresour Technol 2011, 102:3934-3940.

50. Yuan G, Wong SL: Regulation of groE expression in Bacillus subtilis the involvement of the $\sigma^{A}$-like promoter and the roles of the inverted repeat sequence (CIRCE). J Bacteriol 1995, 177:5427-5433.

doi:10.1186/1475-2859-11-101

Cite this article as: $L i$ et al: Rational improvement of the engineered isobutanol-producing Bacillus subtilis by elementary mode analysis. Microbial Cell Factories 2012 11:101. 